\title{
Two patients with balanced translocations and autistic disorder: CSMD3 as a candidate gene for autism found in their common 8 q23 breakpoint area
}

\author{
Chiara Floris $^{1,4}$, Stefania Rassu ${ }^{2,4}$, Loredana Boccone ${ }^{3}$, Daniela Gasperini ${ }^{3}$, Antonio Cao ${ }^{1}$ \\ and Laura Crisponi ${ }^{*}, 1$
}

\begin{abstract}
${ }^{1}$ Istituto di Neurogenetica e Neurofarmacologia INN-CNR, Cagliari, Italy; ${ }^{2}$ Dipartimento di Scienze Biomediche e Biotecnologie, Università degli Studi di Cagliari, Cagliari, Italy; ${ }^{3}$ Ospedale Regionale per le Microcitemie, Clinica Pediatrica II, Azienda U.S.L. 8 Cagliari, Cagliari, Italy
\end{abstract}

Recent studies estimated a rate of 3-5\% of cytogenetic abnormalities involving many different chromosomes in autistic spectrum disorders (ASDs). Here, we report on two unrelated male patients with de novo translocations, autistic behaviour and psychomotor delay. These two patients carry a balanced chromosome translocation $\mathrm{t}(5 ; 8)(\mathrm{q} 14.3 ; \mathrm{q} 23.3)$ and $\mathrm{t}(6 ; 8)(\mathrm{q} 13 ; \mathrm{q} 23.2)$, respectively. A detailed physical map covering the regions involved in the translocations was constructed using BAC clones mapping on chromosomes 5q14.3, 6q13 and 8q23. Fluorescence in situ hybridisation (FISH) analyses were carried out using these genomic clones. We fine mapped the two translocation breakpoints on chromosomes 8 identifying their position within a short $5 \mathrm{Mb}$ genomic region. Breakpoints on chromosomes 8 in both patients do not interrupt any known gene but both map in a region containing the CSMD 3 gene, which thereby can be considered as a candidate for ASDs.

European Journal of Human Genetics (2008) 16, 696-704; doi:10.1038/ejhg.2008.7; published online 13 February 2008

Keywords: autistic spectrum disorder; balanced translocation; FISH; 8q23; CSMD3; position effect

\section{Introduction}

Autistic spectrum disorders (ASDs; MIM 209850) are a group of neurodevelopmental disorders characterised by qualitative impairments in a triad of behavioural domains: (1) social interactions; (2) development of language, communication and imaginative play; (3) restricted, stereotypical and repetitive patterns of interest, behaviour and activities (DMSIV; ICD10). ${ }^{1,2}$ ASDs are relatively common conditions that manifest in early childhood with

\footnotetext{
*Correspondence: Dr L Crisponi, Istituto di Neurogenetica e Neurofarmacologia (INN), Consiglio Nazionale delle Ricerche, c/o Cittadella Universitaria di Monserrato, 09042 Monserrato, Cagliari, Italy.

Tel: + 39070 6754591; Fax: + 39070 6754652;

E-mail: laura.crisponi@inn.cnr.it

${ }^{4}$ These authors contributed equally to this work.

Received 3 August 2007; revised 20 December 2007; accepted 6 January 2008; published online 13 February 2008
}

onset before 36 months of age and are frequently accompanied by mental retardation (75\%) and epilepsy $(25 \%) .{ }^{3}$ Individuals with ASDs are often divided into two groups such as a syndromic or complex group in whom autism is associated by malformations or dysmorphic features and a non-syndromic group where the patients have a normal appearance. ${ }^{4}$

Prevalence of autism estimates in the general population has gradually increased over the last 20 years; ${ }^{5}$ epidemiological studies in North America and Europe report an incidence of about 1 in 150 children with male/female ratio of approximately 4:1.,6 Current evidence suggests that ASDs are predominantly hereditable disorders; heritability is estimated to be more than 90\% genetic and several genes are thought to contribute to the disorder, although the mode of inheritance is unknown. ${ }^{5}$ The rate of recurrence in siblings of affected children is estimated to be 
$2-8 \%$, much greater than the prevalence rate in the general population. ${ }^{1}$ Moreover, twin studies report a rate of concordance of $60-91 \%$ in monozygotic twins (MZ). ${ }^{5}$ Surprising disparity in some MZ twins indicates that other factors can modify these phenotypes. The study of several cases shows that susceptible genetic background and random environmental events may be necessary for the full expression of the disorder. Viral infections, for example, rubella and cytomegalovirus, during or after pregnancy have been associated with ASDs in some infants. Another possible mechanism is a random epigenetic mutation in early embryonic life that altered the expression of the genetic trait. ${ }^{7,8}$

Despite the abundance of investigations into the genetics of ASDs, the identity and number of genes involved are not yet known. The wide phenotypic variability of the ASDs most likely reflects the interaction of multiple genes within an individual's genome and the existence of distinct genes and gene combinations among those affected.

Until recently, only $5-10 \%$ of autism cases were distinguishable with an underlying genetic cause. Two recent studies of Jacquemont $e t a l^{9}$ and Sebat et $a l^{6}$ suggest that this number is actually $10-20 \%$ and that it may grow to $30-40 \%$ with further research. ${ }^{4}$

The identification of the candidate genes for autism through linkage and association studies is very difficult towing to the considerable genetic and phenotypical heterogeneity. Many whole-genome analyses, linkage and association studies in multiplex families have identified genomic regions most likely to contain ASDs susceptibility loci on 20 different chromosomes; regions implicated in different studies include chromosomes $1 \mathrm{q}, 2 \mathrm{q}, 4 \mathrm{q}, 5 \mathrm{q}, 6 \mathrm{q}$, 7q, 8q22-24, 10q, 13q, 15q, 17q, 18q, 22q and X. ${ }^{10-13}$

Several candidate gene studies have been carried out, suggesting the involvement of genes essential in neurodevelopment, synaptic function, language and metabolism (PIK3CG ${ }^{5,14}$ FOXP,${ }^{1,15}$ GABA-A receptor, ${ }^{1,5,16}$ UBE3A, ${ }^{5}$ etc). More recently, mutations in MECP2, PTEN, SHANK and NLGN4 have been reported to be involved in a small percentage of autism cases. ${ }^{17}$

An alternative and potentially successful approach aimed at eliciting candidate genes or candidate regions is the detailed analysis of the boundaries of the cytogenetic abnormalities found in individuals with autism. ${ }^{18}$ Recent studies estimated a rate $>5 \%$ of cytogenetic abnormalities (including unbalanced translocations, inversions, rings and interstitial deletions and duplications) in ASDs, and a high number of such cases have been described in the literature with regard to most chromosomes. ${ }^{5,19,20}$ Two studies $^{6,9}$ greatly strengthen the growing awareness that a substantial fraction of ASDs is caused by genomic rearrangements.

We have identified two male patients with autism and psychomotor delay, each associated with a de novo balanced translocation, respectively $\mathrm{t}(5 ; 8)(\mathrm{q} 14.3 ; \mathrm{q} 23.3)$ and $\mathrm{t}(6 ; 8)(\mathrm{q} 14 ; \mathrm{q} 23.2)$. We report here the fine physical mapping of the breakpoints involved in the two translocations by fluorescence in situ hybridisation (FISH) analysis. This analysis focused on a common area of breakage on the two translocated chromosomes 8 , in the region q23, already observed as a potential susceptibility locus. ${ }^{21}$ The purpose of this study is to search for a new potential susceptibility gene for ASDs in these patients.

\section{Materials/subjects and methods Cytogenetic analysis}

Metaphase slides were prepared from peripheral blood lymphocyte cultures from the two patients, obtained after informed consent, using standard methods. Chromosome analysis was performed by routine QFQ-banding (approximately 550 band resolution). The investigation was extended to their parents who were reported to be normal.

\section{FISH analysis}

FISH experiments with different BAC clones from each rearranged chromosomal region were performed. BAC clones from human contig RPCI-11 were used. BAC DNA was labelled with digoxigenin-11-dUTP using nick translation kit (Roche, Basel, Switzerland). The labelling procedure was carried out according to the manufacturer's instructions. The DNA probe was dissolved in $10 \mu \mathrm{l}$ of hybridisation solution according to the procedure described by Rocchi et al (http://www.biologia.uniba.it/rmc). Digoxigenin-labelled probes were visualised with antibodies anti-mouse and anti-rabbit IgG conjugate. Chromosomes were counterstained with DAPI or propidium Iodide. The slides were analysed in an Olympus fluorescence microscope (BX60), and images were captured by a cooled CCD camera (Sensys) and analysed using an imaging system with MacProbe software v. 4.2.3 (PSI).

\section{Contigs}

We have used the NCBI (http://www.ncbi.nlm.nih.gov/; updated 17 April, 2007), ENSEMBL (http://www.ensembl. org/index.html; Ensembl release 44 - April 2007) and UCSC (http://genome.ucsc.edu; update March 2006) data sets to construct the map, which contains the translocation breakpoints, to search for candidate genes and to assemble figures for this paper.

BAC clones from library RPCI-11 were donated by the Italian Telethon service (San Raffaele, Milano) and by Professor M Rocchi, DI.GE.MI. - Department of Genetics and Microbiology, University of Bari.

\section{Expression analysis}

Total RNA was extracted from the peripheral blood leukocyte of a control sample using the Trizol Reagent (Invitrogen, Carlsbad, CA) according to the manufacturer's recommendations. 
RT-PCR was carried out with the First-Strand cDNA Synthesis kit for RT-PCR (AMV; Roche) according to the manufacturer's instructions. Specific primers for CSMD3 with forward (5'-TGGTCATGAAGTATTTCTTCAGTG-3') and reverse (5'-CATTGATATACAACTGTGTCTCTAT- $\left.3^{\prime}\right)$ directions were designed according to the sequence of the gene (http://www.ncbi.nlm.nih.gov/). First round RT-PCR was performed in $20 \mu \mathrm{l}$ volumes on $800 \mathrm{ng}$ of total RNA. A second round PCR was carried out using $2 \mu \mathrm{l}$ of the first PCR product as template and the specific primers. The amplified products were separated on $2 \%$ agarose gel and visualised by ethidium bromide staining.

\section{Results}

We report here on two unrelated male patients, each with a de novo balanced translocation. The first patient carries $\mathrm{t}(5 ; 8)(\mathrm{q} 14.3 ; \mathrm{q} 23.3)$ and the second $\mathrm{t}(6 ; 8)(\mathrm{q} 13 ; \mathrm{q} 23.2)$. Both are affected by autistic disorder, developmental delay and have a common area of breakage in $8 \mathrm{q} 23$. In addition, the first patient presents with epilepsy also. We initially focused our attention on this common area of breakage in 8q23 to determine if a single gene, possibly interrupted by the translocation, could be the cause of both phenotypes.

\section{Cases report}

Patient 1 (M.L.) A 5.5-year-old Sardinian boy was born to a 31-year-old mother and 36-year-old father, both healthy and nonconsanguineous. There was a history of complicated pregnancy - a funicular knot at the third month, oligohydramnios since the fifth month and fetal growth retardation at the 32nd week of pregnancy. He was delivered by caesarean section at the 34th week of pregnancy. Birth weight was $1540 \mathrm{~g}$ (3rd centile), length was 42.5 (3rd centile) and CC was $29 \mathrm{~cm}$ (3th centile). APGAR scores were 9 and 9 after $1 \mathrm{~min}$ and $5 \mathrm{~min}$, respectively. Brain ultrasonography in the neonatal period showed calcifications in the thalamus and nucleus dentatus bilaterally. The patient showed delayed psychomotor development with an IQ level of 38, severe speech delay, short attention span and epilepsy. The autism evaluation using CARS and ABC manuals met the DMS-IV criteria for autistic disorder. The physical examination at 5.5 years of age showed: height $97 \mathrm{~cm}$ (<3rd centile), weight $15400 \mathrm{~kg}$ (3rd centile) and CC $48.5 \mathrm{~cm}$ (3rd centile). He presented with a wide-based gait, right hemiparesis, stereotypic hand movements and no social interest. Metabolic screening, lysosomal enzyme analysis, fragile $\mathrm{X}$ and Rett DNA testing, ocular and cardiac evaluations as well as PEV, PAE and EMG were normal. A brain MRI showed periventricular leukomalacia more prevalent in the left cerebral hemisphere and the EEG showed multifocal, parossistic and polymorphic anomalies, especially in the anterior cerebral area. The karyotype was $46, \mathrm{XY}, \mathrm{t}(5 ; 8)(\mathrm{q} 14.3 ; \mathrm{q} 23.3)$ de novo.
Patient 2 (A.A.)

A 3.3-year-old Sardinian boy was born at term after an uncomplicated pregnancy. The mother was 22 years old at the time of delivery and the father was 51 years. Consanguinity of IV degrees was noted. His younger sister was healthy. Birth weight was $4200 \mathrm{~g}$ (90th centile), length $52 \mathrm{~cm}$ (90th centile), CC $36.5 \mathrm{~cm}$ (75th centile). He showed early hypotonia and development delay. He walked at 16 months and presented with severe speech delay. At present, he does not talk and he has an IQ score within the moderate range of mental retardation. Autistic behaviour was recognised during childhood with complete avoidance of eye contact and no interest in social relations. He had a short attention span and did not exhibit imaginative play. He met the DSM-IV criteria for autistic disorder using CARS and $\mathrm{ABC}$ manuals for autism evaluation. At physical evaluation, at the age of 3.3 years, he was $99 \mathrm{~cm}$ tall (50th centile) and weighed $17 \mathrm{~kg}$ (75th centile) with a CC of $50 \mathrm{~cm}$ (40th centile). He showed joint hypermobility, and high-arched palate but did not display dimorphic features. Metabolic screening, fragile $X$ and Rett DNA testing, EEG, brain MRI scan, ocular and cardiac evaluations and PAE were normal. The karyotype was 46,XY,t(6;8)(q13;q23.2) de novo.

\section{Mapping of the 8q23 translocation breakpoints}

To localise the breakpoints of both translocations $t(5 ; 8)$ and $t(6 ; 8)$, first on chromosomes 8 , we performed a systematic series of FISH analyses using BAC clones from the RPCI-11 human genomic BAC library mapping to the $8 q 23$ region. We constructed a BAC contig map with ENSEMBLE and UCSC databases. The BAC clones RP11536K17 and RP11-118J9 in the patient $\mathrm{t}(5 ; 8)$ (Figure 1a and c) and the BAC clone RP11-946L20 in the patient $t(6 ; 8)$ (Figure $1 \mathrm{~b}$ and $\mathrm{c}$ ), respectively, spanned the chromosome 8 breakpoint. To identify a candidate gene mapping to the two common breakpoint regions, a genomic sequence analysis was performed by searching the NCBI, ENSEMBLE and UCSC databases.

In both patients, no known gene is interrupted by the translocation breakpoints on chromosomes 8 . In patient 1 , the breakpoint occurs $\sim 2.8 \mathrm{Mb}$ distal to the $5^{\prime}$ of the CSMD3 gene and $\sim 0.5 \mathrm{Mb}$ distal to the $5^{\prime}$ of the TRPS1 gene; in patient 2 , the breakpoint is located $\sim 1.1 \mathrm{Mb}$ distal to the $3^{\prime}$ end of the CSMD3 gene (Figure 1c). Both chromosomes 8 breakpoints are close to CSMD3 (CUB and Sushi multiple domains 3), a gene spanning on $1.2 \mathrm{Mb}$ in 8 q23 consisting of 73 exons. ${ }^{22,23}$ This gene encodes a transmembrane protein composed of a long N-terminal polypeptide with CUB and Sushi multiple domains exposed outside the cell, a single transmembrane domain and a C-terminal domain of 55 amino-acid residues located in the cytoplasm. Northern blot hybridisation using human adult and fetal multiple-tissue blots and PCR amplification of different cDNA fragments using a 

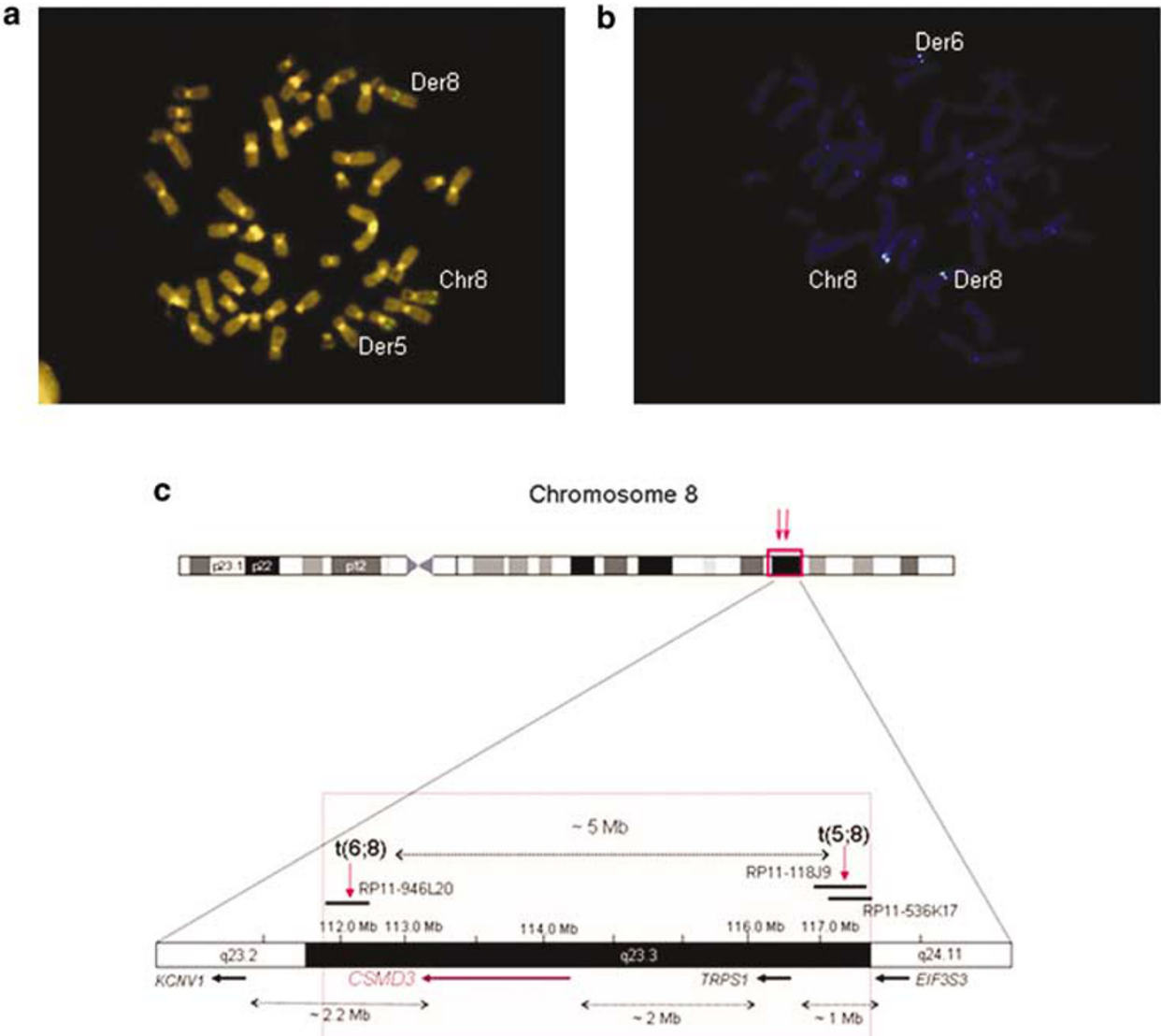

Figure 1 Mapping of the $8 \mathrm{q}$ breakpoint regions involved in the $\mathrm{t}(5 ; 8)$ and $\mathrm{t}(6 ; 8)$ translocations using chromosome 8 BAC clones. (a) Fluorescent in situ hybridisation (FISH) analysis with the clone RP11-536K17 on metaphase chromosomes of patient 1. Hybridisation signals (green dots) can be detected on normal chromosome 8 (chr 8) and the two derivate chromosomes der(5) and $\operatorname{der}(8)$. (b) FISH analysis with the clone RP11-946L20 on metaphase chromosomes of patient 2. Hybridisation signals (white dots) can be detected on the normal chromosome 8 (chr 8 ) and the two derivate chromosomes der(6) and der(8). (c) Physical map of the 8q23 region with corresponding genomic BAC clones used for FISH and map of the region surrounding the two breakpoints on chromosome 8. Arrows indicate the transcription orientation of the genes CSMD3 and TRPS1 relative to the centromere of chromosome 8 and the location of the breakpoints (red). The diagram is not drawn to scale.

multiple-tissue cDNA panel were performed by Shimizu et $a l^{22}$ and the analyses indicated that the CSMD3 gene is weakly expressed in most tissues, while it is expressed mainly in the adult and fetal brain and testis as a transcript of about $13 \mathrm{~kb}$. Some shorter transcripts were also found in the testis and fetal brain. Any CSMD3 transcript was detected in leukocytes. ${ }^{22}$ To verify the expression of the gene on peripheral blood, we performed an RT-PCR on three control samples but never detected any transcript (data not shown). To exclude the possibility of an overexpression of CSMD3 led by the rearrangements, we verified its expression in the leukocytes of translocated patient 2. No transcript was detected (data not shown). Comparative genomic analysis revealed the presence of a member belonging to the conserved family of CSMD3 in the mouse and fugu genomes.

The other gene closer to the translocation breakpoint in patient 1 is TRPS1 (trichorhinophalangeal syndrome I), a gene that encodes for a 1281 amino-acid-specific zinc-finger protein, which is a putative transcription factor. $^{24,25}$ Mutations in this gene lead to autosomal dominant disorders characterised by craniofacial and skeletal anomalies - the trichorhinophalangeal syndromes (TRPS). Three variants of this syndrome have been described in the literature: TRPSI (MIM 190350) and TRPSII, also known as Langer-Giedion syndrome (MIM 150230) and TRPSIII (MIM 190351). The hallmarks found in all three types are sparse scalp hair, a bulbous tip of the nose, bushy eyebrows, a long flat philtrum, a thin upper vermilion border, protruding ears and short stature. Skeletal abnormalities include cone-shaped epiphyses at the phalanges, hip malformations and short stature. TRPSII is an example of contiguous gene syndromes: patients with TRPSII, or Langer-Giedion syndrome, have also multiple cartilaginous exostoses caused by mutations in the EXT1 gene, which is mutated in a subgroup of patients with multiple cartilaginous exostoses and deleted in all patients with TRPS type II. 
Analysis of the $8 \mathrm{q} 23$ breakpoints area by the Ensemble database DECIPHER (http://decipher.sanger.ac.uk/) and by the Autism chromosomal rearrangements database (http:// projects.tcag.ca/autism/; update 18 May 2007) showed that there are no other chromosomal rearrangements or segmental duplications reported so far associated with ASDs.

Sebat et $a l^{6}$ showed that copy number variations (CNVs) are more frequent in patients with ASDs than in unaffected individuals and more frequent in sporadic cases than in 'multiplex' families. We carried out a more detailed analysis of this region using the database of the Autism CNV Project (http://projects.tcag.ca/cgi-bin/naar10k/ cnvHome). This database reports the results of a recent study on autism genetics performed using an analysis of $\mathrm{CNV}$ in families with ASDs (Autism Genome Project Consortium, 2007). This analysis showed the presence of $8 \mathrm{CNVs}$ (all gain) associated with autism overlapping the 8 q23 breakpoint region harbouring the CSMD3 gene. We performed the same analysis using the database of genomic variants (http://projects.tcag.ca/variation) and ascertained that in the same region, there are 20 genomic variations reported in the normal population.

Furthermore, a detailed analysis of the 8q23 breakpoints area by the Ensemble database showed that noncoding RNAs are not located in this $\sim 5 \mathrm{Mb}$ region and that, in addition, there are LINE and SINE transposones and recombination hot spot.

a

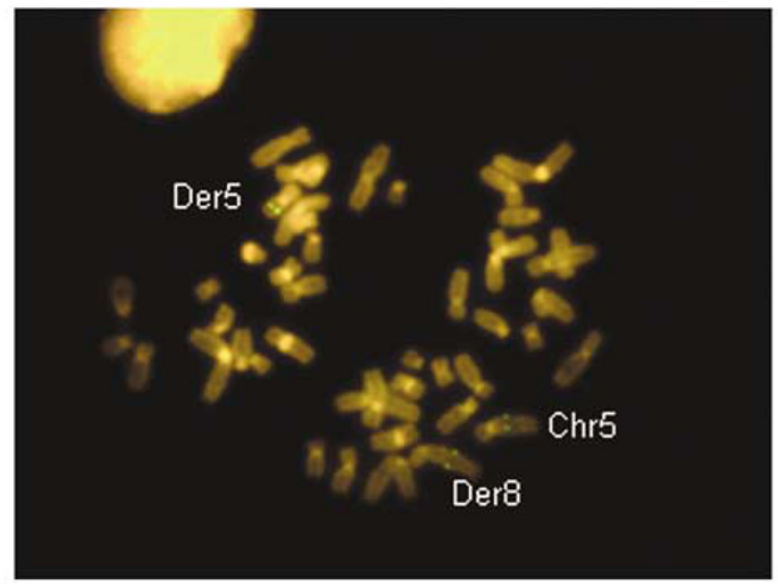

b

Chromosome 5

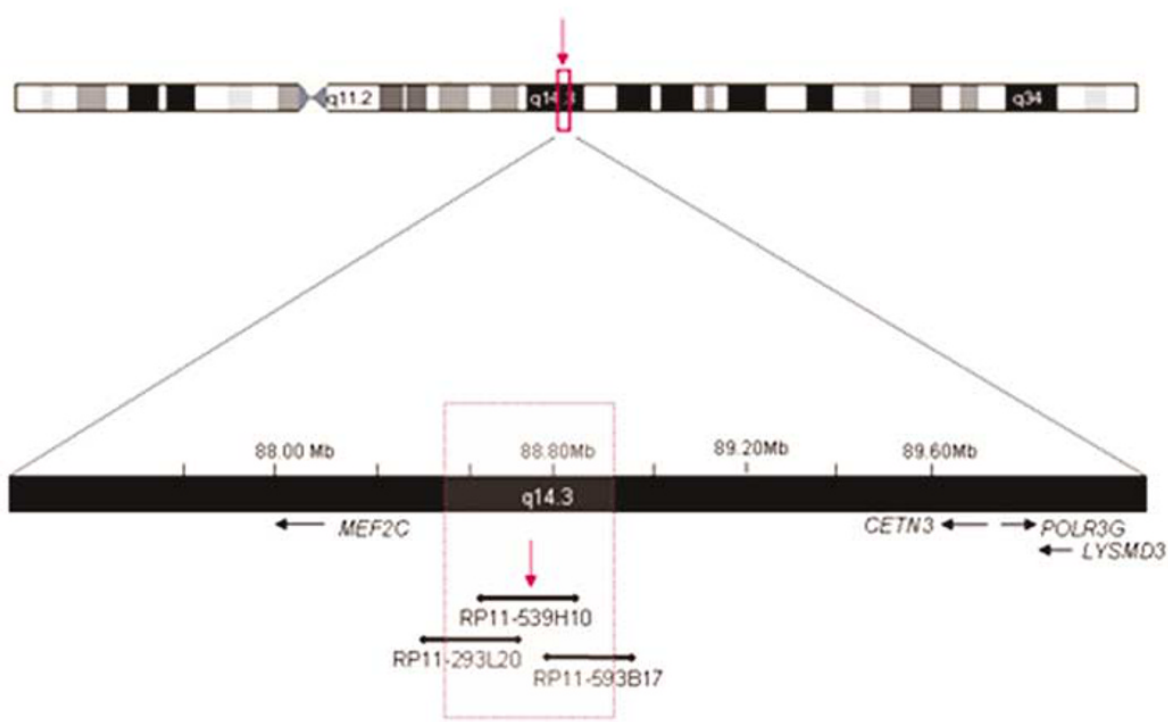

Figure 2 Mapping of the $5 q$ breakpoint region involved in the $t(5 ; 8)$ translocation using chromosome 5 BAC clones. (a) FISH analysis with the clone RP1 1-539H10 on metaphase chromosomes. Hybridisation signals (green dots) can be detected on normal chromosome 5 (chr 5 ) and the two derivate chromosomes der(5) and der(8). (b) Physical map of the 5q14.3 region with corresponding genomic BAC clones used for FISH and map of the region surrounding the breakpoint on chromosome 5. Arrows indicate the transcription orientation of the genes relative to the centromere of chromosome 5 and the location of the BAC clones. The diagram is not drawn to scale. 
Mapping of the 5q14.3 breakpoint

The breakpoint on chromosome 5 was also characterised by FISH experiments using clones from the RPCI-11 human genomic BAC library. The BAC clone RP11-539H10 spanned the chromosome 5 breakpoint (Figure 2a and b). A genomic sequence analysis was performed by searching the public databases: also, in this case, no known gene was interrupted by the chromosome rearrangement.

The gene found proximal to the translocation is MEF2C (myocyte enhancer factor 2C): this regulatory protein is, like the myogenic basic helix-loop-helix proteins, involved in myogenesis. The KO mouse for this gene showed that its transcript is an essential regulator of cardiac morphogenesis and right ventricular development. ${ }^{26}$ Variants of MEF2C were found in the skeletal muscle and brain. Leifer $e t a l^{27}$ found that the brain form was expressed by neurons in particular layers of the cerebral cortex and that expression declined during post-natal development. The gene distal to the breakpoint is CETN3 (centrin, EF-hand protein, 3): the protein encoded by this gene contains four EF-hand calcium-binding domains, and it is a member of the centrin protein family; this was highly enriched in the centrosome fraction of cell extracts and appears to represent the most abundant centrosomal isoform. ${ }^{28}$

In this chromosomal region, the analysis by DECIPHER showed that there are three other chromosomal rearrangements associated with mental retardation/developmental delay and some other minor features:

- 46, XY, del(5)(q14.3;q14.3);

- 46, XY, dup(5)(q13.3;q15).
Furthermore, the Ensemble database shows that noncoding RNAs are not located in this area. We carried out a more detailed analysis using the database of the Autism $\mathrm{CNV}$ Project that showed the presence of five CNVs overlapping this region between the $M E F 2 C$ and the CETN3 genes and we found out that there are three genomic variants also reported in the normal population (http://projects.tcag.ca/variation).

\section{Mapping of the $6 \mathrm{q} 13$ breakpoint}

Breakpoint mapping was carried out by FISH analysis with BAC probes from the chromosomal region 6q13. The BAC clones delimiting the chromosomes 6 breakpoint were RP11-678H04 and RP11-453A07. It was not possible to locate the fine position of the breakpoint because we no longer have the availability of the blood sample for patient 2 . The genomic region included between the telomeric and centromeric BAC clones is $\sim 0.7 \mathrm{Mb}$ and there is no known gene interrupted by the translocation breakpoint (Figure 3).

The gene closer to the translocation is CD109 (a GPIlinked cell-surface antigen) ${ }^{29}$ and the distally located gene is COL12A1 (collagen, type XII, $\alpha 1$; this gene encodes the $\alpha$ chain of type XII collagen, a member of the FACIT - fibrilassociated collagens with interrupted triple helices collagen family). ${ }^{30}$ Another gene next to the translocation breakpoint is SLC17A5 (solute carrier family 17, member 5): mutations of this gene cause Salla disease (adult form of sialuria, also called sialic acid storage disease) characterised by progressive psychomotor retardation of early onset, lysosomal storage and increased urinary excretion of free sialic acid. ${ }^{31}$ Analysis by using the Ensemble database

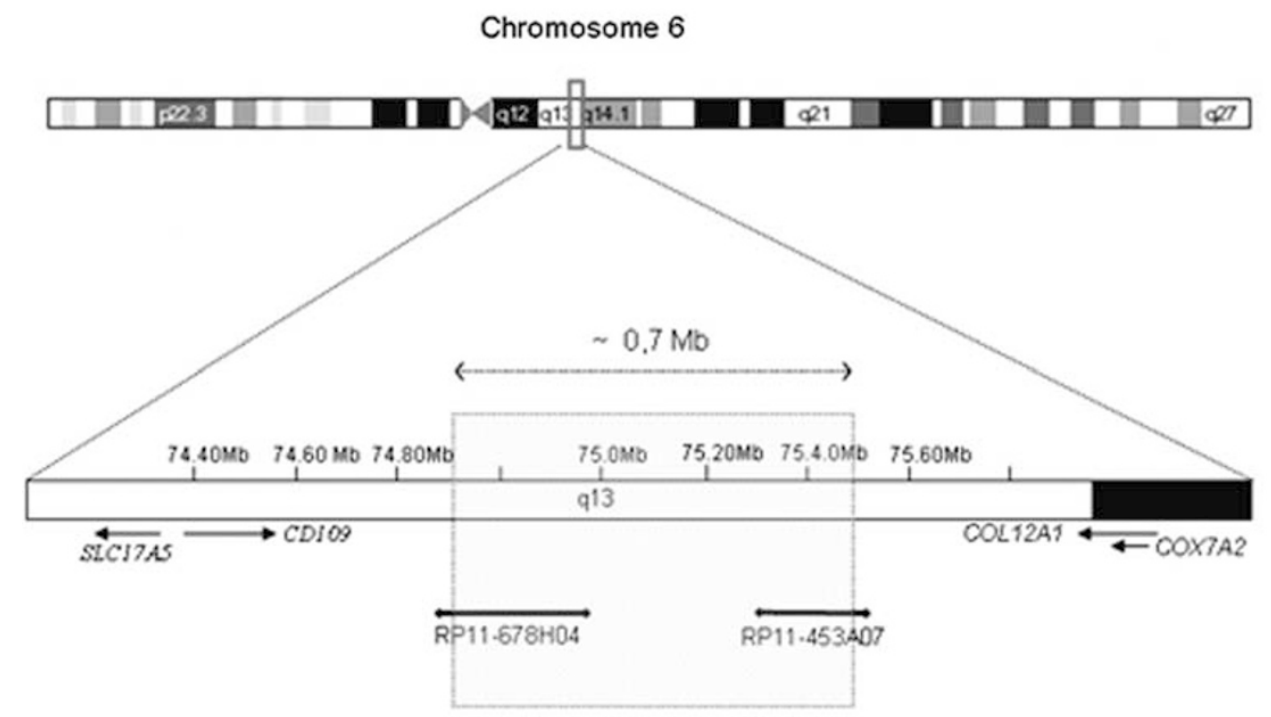

Figure 3 Physical map of the $6 \mathrm{q} 13$ region with corresponding genomic BAC clones used for FISH and map of the region surrounding the breakpoint on chromosome 6 . Arrows indicate the transcription orientation of the genes relative to the centromere of chromosome 6 and the location of the 2 BAC clones. The diagram is not drawn to scale. 
(DECIPHER) and the Autism Chromosome Rearrangement Database of this chromosomal region revealed other more chromosomal rearrangements characterised by mental retardation/developmental delay, autism and some other minor features:

- $\operatorname{del}(6)(\mathrm{q} 13 ; \mathrm{q} 14.1)$

- $\operatorname{del}(6)(\mathrm{q} 13 ; \mathrm{q} 16.1)$

- $\mathrm{t}(6 ; 14)(\mathrm{q} 13 ; \mathrm{q} 21)$.

Detailed analysis of this 6q13 breakpoint region performed with both the database of the Autism CNV Project and the database of genomic variants showed the presence of $7 \mathrm{CNVs}$ and 6 genomic variations, respectively. A detailed analysis of this area by the Ensemble database pointed out that noncoding RNAs are not located in this region.

\section{Discussion}

The study of chromosomal rearrangements associated with abnormal phenotypes has proven to be a powerful method for the identification of disease-related genes. Indeed, there are numerous examples of human disorders in which analysis of chromosomal abnormalities has subsequently led to the discovery of critical disease intervals and eventually to the identification of genes involved in the aetiology of a disease. ${ }^{32-34}$ In this paper, we report two unrelated male patients with balanced reciprocal translocations $\mathrm{t}(5 ; 8)(\mathrm{q} 13 ; \mathrm{q} 23.3)$ and $\mathrm{t}(6 ; 8)(\mathrm{q} 13 ; \mathrm{q} 23.2)$, associated with ASDs and developmental delay. Both patients were reported to have a common breakage area in 8q23.

FISH analyses with BAC clones were carried out to determine precisely the position of each chromosomal breakpoint to identify candidate gene(s) implicated in the pathogenesis of autistic disorder. To find the candidate gene, a genomic sequence analysis was performed by searching the public databases (NCBI, ENSEMBL and UCSC). This analysis excluded the presence of known genes interrupted by these breakpoints and, moreover, showed that on chromosomes 8 both breakpoints are located in a $5 \mathrm{Mb}$ genomic region between BAC RP11946L20 and RP11-536K17, region that contains the CSMD3 gene.

Although the hypothesis of a 'disease gene at breakpoint' would be the simplest one, more and more evidence is being accumulated to support the existence of different mechanism underlying disease with chromosomal rearrangements outside genes and their regulatory regions known as 'position effect'. ${ }^{35,36}$ The mechanism that may cause such effects are: (1) separation of the gene from its enhancer or promoter region, (2) removal of the longrange insulator or boundary element, (3) competition with another enhancer, (4) juxtaposition with an enhancer element from another gene and (5) position-effect variegation, that is the insertion of the gene in a new heterochromatin environment.

Considering the positional effect, our observations suggest that CSMD3 is a positional candidate gene for ASDs. The literature reports that this chromosomal region harbours a genetic locus for the benign adult familiar myoclonic epilepsy type 1 (BAFME1/GFAME; MIM 601068). ${ }^{37}$ Mutational analysis of the CSMD3 gene in patients with BAFME1/GFAME identified three single nucleotide changes that are not responsible for the pathogenesis of this syndrome. ${ }^{22}$

The expression of CSMD3 on the fetal and adult brain suggests that this gene is a good candidate for the pathogenesis of ASDs. There is little information about the function of CUB - containing proteins, although it has been postulated that they would be mainly involved in the developmental process and that CUB and Sushi domains are thought to be sites of protein-protein or proteinligand interactions, suggesting that CSMD3 proteins are either transmembrane receptors or adhesion proteins. A recent study shows that SEZ- 6 containing both CUB and Sushi domains is involved in signalling and cell-cell adhesion. SEZ-6 is strongly expressed in the developing forebrain and is necessary for the formation of the neural network. ${ }^{23}$

CSMD1 (CUB and Sushi multiple domain 1), the founding member of the gene family containing multiple CUB and Sushi multiple domain, has been cloned as a candidate tumour-suppressor or tumour-progression gene associated with head and neck squamous cell carcinomas and in prostate cancers. Richter et $a l^{38}$ showed that promoter methylation of CSMD1 might epigenetically silence the gene; particularly, they suggest that methylation of the DNA preceding CSMD1's first exon is correlated with the downregulation of its level of expression in the squamous cell carcinoma cell lines; whereas in the normal tissue, there is little promoter methylation. In the light of these observations, we think that it would be interesting to also evaluate the methylation status for CSMD3.

These findings should encourage further investigations, including the mutational screening of CSMD3 in a large group of patients with ASDs, to clarify an association with this pathology and its potential role in cognitive function.

The other gene closer to the breakpoint region on chromosomes 8 in patient 1 is TRPS1. Mutations of this gene lead to an autosomal dominant disorder, the trichorhinophalangeal syndromes (TRPS), characterised by craniofacial and skeletal anomalies, ${ }^{24}$ which were not observed in our patients. Although the $8 \mathrm{q} 23$ region is the most likely location for an ASDs autism candidate gene for each of the cases described here, the possibility that the phenotype observed in the patients is a consequence of a gene defect occurring at the breakpoint on chromosomes 5 and 6 , respectively, cannot be ruled out. It is generally 
accepted that most neuropsychiatric development disorders like ASDs are polygenic or multifactorial; that is, several synergistically acting genes in an affected individual's genome may be required to produce the full autistic phenotype. Furthermore, brain development and complex behaviours are multidetermined, with genes turning cascades of proteins on or off, while they influence one another. A specific set of genetic polymorphisms may determine one's susceptibility to ASDs, yet, even then, environmental triggers may modify the phenotypic expression of the disorder. In these patients, we suppose that changes in the dosage of genes could have quantitative effects on gene functions, including haploinsufficiency or altered transcription patterns; instead, we cannot exclude the involvement of epigenetic regulatory mechanisms in the aetiology of the autism, as suggested in other cases. ${ }^{7}$

Our observations regarding the expression of CSMD3 in fetal and adult brain suggest that this gene may be involved in the pathogenesis of ASDs. However, future studies, including mutational analysis of CSMD3 in a large number of patients affected by autism and behavioural studies on a Csmd3 knockout mouse, are necessary to confirm or reject this assumption.

\section{Acknowledgements}

We thank the patients and their families for their contribution to this study. We thank Manuela Uda for the critical reading of the manuscript. We would like to acknowledge Professor M Rocchi and Italian Telethon service (San Raffaele, Milano) for the BAC clones service. We dedicate this article to the memory of Paol Giuseppe Pilie.

\section{References}

1 Muhle R, Trentacoste SV, Rapin I: The genetics of autism. Pediatrics 2004; 113: 472-486.

2 American Psychiatric Association: DSM-IV - Diagnosis and Statistical Manual of Mental Disorders 1994, (4th edn) American Psychiatric Publishing: Washington DC.

3 Ishikawa-Brush Y, Powell JF, Bolton P: Autism and multiple exostoses associated with an X;8 translocation occurring within the GRPR gene and $3^{\prime}$ to the SDC2 gene. Hum Mol Genet 1997; 6: $1241-1250$.

4 Beaudet AL: Autism: highly heritable but not inherited. Nat Med 2007; 13: 534-536.

5 Vorstman J, Staal WG, van Daalen E, van Engeland $H$, Hochstenbach PFR, Franke L: Identification of novel autism candidate regions through analysis of reported cytogenetic abnormalities associated with autism. Mol Psychiatry 2006; 11: $18-28$.

6 Sebat J, Lakshmi B, Malhotra D et al: Strong association of de novo copy number mutations with autism. Science 2007; 316: 445-449.

7 Schanen NC: Epigenetics of autism spectrum disorders. Hum Mol Genet 2006; 15: 138-150.

8 Badcock C, Crespi B: Imbalanced genomic imprinting in brain development: an evolutionary basis for the aetiology of autism. J Evol Biol 2006; 19: 1007-1032.

9 Jacquemont ML, Sanlaville D, Redon R et al: Array-based comparative genomic hybridisation identifies high frequency of cryptic chromosomal rearrangements in patients with syndromic autism spectrum disorders. J Med Genet 2006; 43: 843-849.
10 Yonan AL, Alarcòn M, Cheng R et al: A genomewide screen of 345 families for autism-susceptibility loci. Am J Hum Genet 2003; 73: 886-897.

11 Freitag CM: The genetics of autistic disorders and its clinical relevance: a review of the literature. Mol Psychiatry 2007; 12: 2-22.

12 Herbert MR, Russo JP, Yang $S$ et al: Autism and environmental genomics. Neurotoxicology 2006; 27: 671-684.

13 Philippe A, Martinez M, Guilloud-Bataille M et al: Genome-wide scan for autism susceptibility genes. Hum Mol Genet 1999; 8: $805-812$.

14 Serajee FJ, Nabi R, Zhong H, Mahbubul Huq AH: Association of INPP1, PIK3CG, and TSC2 gene variants with autistic disorder: implications for phosphatidylinositol signalling in autism. J Med Genet 2003; 40: 119.

15 Gong X, Jia M, Ruan Y et al: Association between the FOXP2 gene and autistic disorder in Chinese population. Am J Med Genet B Neuropsychiatr Genet 2004; 127: 113-116.

16 McCauley JL, Olson LM, Delahanty R et al: A linkage disequilibrium map of the $1-\mathrm{Mb} 15 \mathrm{q} 12 \mathrm{GABA}(A)$ receptor subunit cluster and association to autism. Am J Med Genet B Neuropsychiatr Genet 2004; 13: 51-59.

17 Persico AM, Bourgeron T: Searching for ways out of the autism maze: genetic, epigenetic and environmental clues. Trends Neurosci 2006; 29: 349-358.

18 Castermans D, Vermeesch JR, Fryns JP et al: Identification and characterization of the TRIP8 and REEP3 genes on chromosome $10 \mathrm{q} 21.3$ as novel candidate genes for autism. Eur J Hum Genet 2007; 15: 422-431.

19 Castermans D, Wilquet V, Steyaert J, Van De Ven W, Fryns JP, Devriendt K: Chromosomal anomalies in individuals with autism. Autism 2004; 8: 141-161.

20 Reddy KS: Cytogenetic abnormalities and fragile-X syndrome in Autism Spectrum Disorder. BMC Med Genet 2005; 6: 1-16.

21 Ylisaukko-oja T, Alarcón M, Cantor RM, Auranen M, Vanhala R, Kempas E: Search for autism loci by combined analysis of Autism genetic resource exchange and Finnish families. Ann Neurol 2006; 59: $145-155$.

22 Shimizu A, Asakawa S, Sasaki T et al: A novel giant gene CSMD3 encoding a protein with CUB and sushi multiple domains: a candidate gene for benign adult familial myoclonic epilepsy on human chromosome 8q23.3-q24.1. Biochem Biophys Res Commun 2003; 309: 143-154.

23 Ling Lau W, Scholnick SB: Identification of two new members of the CSMD gene family. Genomics 2003; 82: 412-415.

24 Momeni P, Glockner G, Schmidt O et al: Mutations in a new gene, encoding a zinc-finger protein, cause tricho-rhino-phalangeal syndrome type I. Nat Genetic 2000; 24: 71-74.

25 Ludecke HJ, Wagner MJ, Nardmann J et al: Molecular dissection of a contiguous gene syndrome: localization of the genes involved in the Langer-Giedion syndrome. Hum Mol Genet 1995; 4: 31-36.

26 Lin Q, Schwarz J, Bucana C, Olson EN: Control of mouse cardiac morphogenesis and myogenesis by transcription factor MEF2C. Science 1997; 276: 1404-1407.

27 Leifer D, Krainc D, Yu YT et al: MEF2C, a MADS/MEF2-family transcription factor expressed in a laminar distribution in cerebral cortex. Proc Natl Acad Sci USA 1993; 90: 1546-1550.

28 Middendorp S, Paoletti A, Schiebel E, Bornens M: Identification of a new mammalian centrin gene, more closely related to Saccharomyces cerevisiae CDC31 gene. Proc Natl Acad Sci USA 1997; 94: 9141-9146.

29 Lin M, Sutherland DR, Horsfall W et al: Cell surface antigen CD109 is a novel member of the alpha(2) macroglobulin/C3, C4, C5 family of thioester-containing proteins. Blood 2002; 99: $1683-1691$.

30 Gerecke DR, Olson PF, Koch $\mathrm{M}$ et al: Complete primary structure of two splice variants of collagen XII, and assignment of alpha 1(XII) collagen (COL12A1), alpha 1(IX) collagen (COL9A1), and alpha 1(XIX) collagen (COL19A1) to human chromosome 6q12q13. Genomics 1997; 41: 236-242. 
31 Verheijen FW, Verbeek E, Aula N, Beerens CEMT, Havelaar AC, Joosse M: New gene, encoding an anion transporter, is mutated in sialic acid storage diseases. Nat Genet 1999; 23: 462-465.

32 Crisponi L, Deiana M, Loi A et al: The putative forkhead transcription factor FOXL2 is mutated in blefarophimosis/ptosis/ epicanthus inversus syndrome. Nat genetics 2001; 27: 159-166.

33 Srivastava AK, Montoen O, Saarialho-Kere U et al: Fine mapping of the $E D A$ gene: a traslocation breakpoint is associated with a CpG island that is transcribed. Am J Hum Genet 1996; 1: 126-132.

34 Tadin-Strapps M, Warburton D, Salas-Alanis JC, Lopez-Cepeda LD, Christiano AM: Fishing for new genes in skin biology: impact of cytogenetics on gene discovery. Clin Genet 2004; 66: 94-106.
35 Kleinjan DJ, van Heyningen V: Position effect in human genetic disease. Hum Mol Genet 1998; 7: 1611-1618.

36 Velagaletti GVN, Bien-Willner GA, Northup JK et al: Position effects due to chromosome breakpoints that map $\sim 900 \mathrm{~Kb}$ upstream and $\sim 1.3 \mathrm{Mb}$ downstream of SOX9 in two patients with campomelic dysplasia. Am J Hum Genet 2005; 76: 652-662.

37 Mikami M, Yasuda T, Terao A: Localization of a gene for benign adult familiar myoclonic epilepsy to chromosome 8q23.3-q24.1. Am J Hum Genet 1999; 65: 745-751.

38 Richter MT, Tong BD, Scholnick SB: Epigenetic inactivation and aberrant transcription of CSMD1 in squamous cell carcinoma cell lines. Cancer Cell Int 2005; 5: 29. 